\title{
Politik und Profit
}

\author{
Bei der Analyse von Produkten in ökologischen Nischenmärkten fällt auf, \\ dass viele Produkfe auf Grüne Gründungen zurückgehen. Der folgende Text \\ fokussiert auf Grüne GründerInnen als politisch motivierte Personen und \\ ihre Netzwerke und leitet daraus eine Reihe von Konsequenzen für die \\ Gründungsförderung ab.
}

$\mathrm{D}$ Von Jens Clausen e empirische Basis besteht in einer im Herbst 2002 durchgeführten Befragung von GründerInnen im ökologischen Lebensmittelsektor. Ziel war es, den Werthaltungen und Gründungsmotivationen Grüner GründerInnen näher zu kommen und gleichzeitig etwas über den ökonomischen Erfolg der Aktivitäten in Erfahrung zu bringen. Die per E-Mail durchgeführte Befragung richtete sich an GründerInnen von Unternehmen der Verarbeitung von und des Handels mit ökologischen Lebensmitteln in drei Regionen: den USA, Nordeuropa und Deutschland. Der Fragebogen wurde an insgesamt 203 Unternehmen gesandt, von denen 50 antworteten, was einer Rücklaufquote von 25 Prozent entspricht. Die Ergebnisse dieser empirischen Befragung lassen dabei notwendigerweise eine Lücke dort, wo es um persönliche Entwicklungen oder gar Zufälle geht, die einzelne Personen zu GründerInnen gemacht haben. Auch Netzwerkstrukturen lassen sich so nicht beleuchten. Diese Lücke wurde durch drei Fallstudien geschlossen (1).

\section{Umweltbewegte GründerInnen}

Viele Grüne Gründungen sind in gleich zweifacher Hinsicht eine Folge der Umweltbewegung. Zum einen haben die besonderen Werthaltungen Grüner GründerInnen ihren Ursprung in der Ausbildung ökologischer Werte und Meinungen, die bei etwa zwei Drittel der GründerInnen mit einer politischen Aktivität in der Umweltbewegung einhergingen. Zum anderen sehen Grüne GründerInnen ihre Gründung als Beitrag zu einem gesellschaftlichen Veränderungsprozess (2).

Die GründerInnen dieser Unternehmen zeigen im Vergleich zu Führungspersonen konventioneller Unternehmen deutliche Unterschiede bei umweltbezogenen Meinungen und Werthaltungen. Sie sehen umweltbezogene Probleme als bedeutender an und vertrauen weniger auf technisch-wissenschaftliche Lösungsmöglichkeiten. In ihrem öko- logischen Leitbild haben Konsistenz, das heißt die Harmonisierung der Produkte mit dem natürlichen Stoffkreislauf, und Subsistenz einen hohen Stellenwert. Diesen Typ der ökologisch orientierten GründerIn bezeichnet die Arbeit als „,Grüne GründerIn“ (3).

Hinsichtlich ihrer Motivation und ihrer Erfolgsmaßstäbe unterscheiden sich Grüne GründerInnen deutlich vom neoklassischen Bild des auf Gewinn fokussierten Unternehmers. Es kann gezeigt werden, dass die meisten von ihnen die Erfuillung ihrer Mission - die Förderung der Produktion und des Konsums ökologisch erzeugter Lebensmittel - als wichtiger als persönlichen Gewinn sehen. Ihr Erfolgsmaßstab ist daher eher die realisierte Produktionsmenge und die damit zusammenhängende Verdrängung ,konventioneller“ Ware vom Markt als der Profit. Dies spiegelt sich auch in ihrer Priorität, erzielte Gewinne eher im Unternehmen zu belassen als in Form von Gewinn zu entnehmen. Und Grüne Gründungen fokussieren ihre Arbeit besonders auf die Marktenwicklung. Dies zeigt sich an überdurchschnittlichen Aufwänden für Marketing und Öffentlichkeitsarbeit.

\section{Grüne Netzwerke}

In den Fallstudien zu den drei männlichen Gründern konnte gezeigt werden, dass neben den vorhandenen Kontakten zu konventionellen Institutionen ein differenziertes Netzwerk aus Kontakten zu ökologisch motivierten Akteuren die Gründung grüner Unternehmen erleichtert und in der weiteren Existenz der Unternehmen eine wesentliche Rolle spielt.

Eines der Fallbeispiele beschreibt einen biologischen Pflanzenzïchter, der sowohl auf der Lieferanten- wie auf der Kundenseite eng mit anderen Akteuren der ökologischen Lebensmittelkette vernetzt ist. Aber auch Bezüge zur konventionellen Züchtungsforschung wie auch zur Landwirtschaftsverwaltung sind von hoher Bedeutung. Die drei in den Fallstudien vorgestellten Grünen Gründungen gehören drei unterschiedlichen Produktionsstufen der Lebensmittelwirtschaft an. Die Aktivitäten des Naturkostgroßhändlers umfassen die Lebensmittelverarbeitung und einen Spezialitätengroßhandel. Der Getreidezüchter betreibt ein kleines Zuchtunternehmen. Die Aktivitäten des Schweinezuchtvereins sind zwar ebenfalls auf die Zucht gerichtet, umfassen aber auch die produzierende Landwirtschaft und zielen auf vertikale Integration einer Verarbeitungs- und Vermarktungsstufe.

Die drei Gründer entsprechen in jeweils unterschiedlichen Merkmalen dem Grünen Gründer. So wird deutlich, dass in der Vita aller drei Gründer die Phase des Kontaktes mit ökologischem Gedankengut zeitlich einige Jahre vor der Gründung begann. Zwei der Gründer machten im Interview implizit deutlich, dass ihnen eine nicht-

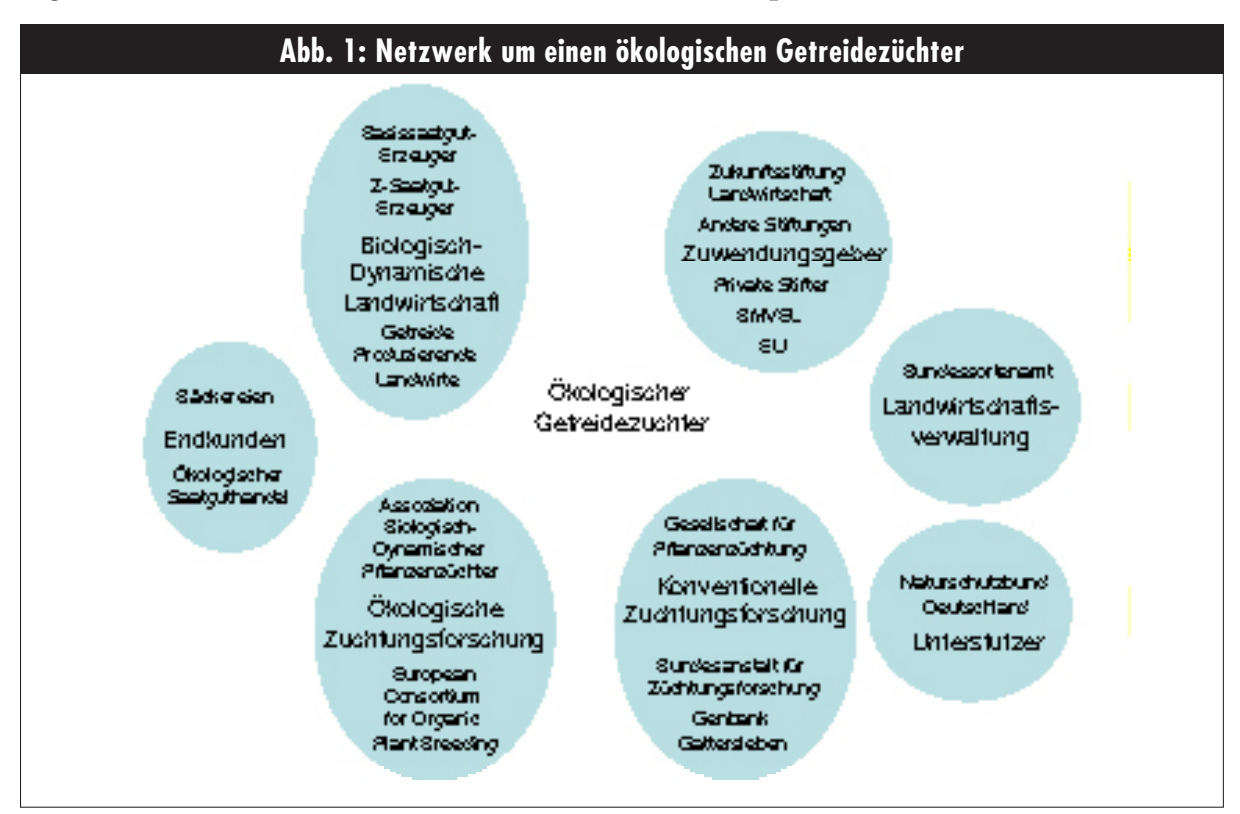


ökologische Gründung zumindest zum Gründungszeitpunkt undenkbar schien. Bei allen drei Gründern ist die politische Absicht untrennbar mit der Gründung verbunden.

Und alle drei weisen auch Merkmale des Entrepreneurship auf. Der Gründer des Naturkostgroßhandels führte zum Beispiel das anfängliche Kollektiv schon drei Jahre nach der Gründung als Eigentümer-Unternehmer weiter und bewies Unternehmergeist und Durchsetzungsvermögen in einer Vielzahl von Aktivitäten auch außerhalb des Unternehmens. Durch das Erkennen von Trends, am Markt orientierte Produktentwicklung und vielfältige Maßnahmen zur Entwicklung des ökologischen Marktsegmentes erreichte er die Festigung seines Unternehmens, welches auch 27 Jahre nach der Gründung floriert.

Die drei Gründer konnten und können ihre Ideen nicht ohne ein verzweigtes Netzwerk von Kontakten zu anderen, ebenfalls ökologisch motivierten Einzelpersonen wie Unternehmen umsetzen. Die beiden auf Zucht gerichteten Gründungen verfügen beispielsweise jeweils über zahlreiche Kontakte in die ökologisch orientierte wie auch in die konventionelle Wissenschaft. Diese beiden Gründungen werden auch in begrenztem Umfang von Umweltverbänden unterstïtzt. Ebenso sind Akteure der staatlichen Landwirtschaftsverwaltung für sie wichtig, in deren Reihen sich vereinzelte ökologisch orientierte Unterstuitzer finden. Eine Besonderheit des Getreidezüchters besteht in einem Kreis privater Zuwendungsgeber rund um die anthroposophische Zukunftsstiftung Landwirtschaft.

Für alle drei Gründungen sind weiter die Existenz eines auf ökologische Produkte gerichteten Marktes und jeweils spezielle der darin aktiven Akteure von Bedeutung. Wesentlich für die zukünftige Entwicklung ist für alle drei Gründungen die Fähigkeit, mit neuen Produkten Zugang zu diesem Markt zu finden. So kann es zum Beispiel, wie im Fall des Schweinezüchters, ein engagierter Landwirt sein, der durch seine Entscheidung, eine seltene Nutztierrasse zu halten, in die Situation kommt, deren Vermarktung in die Hand nehmen zu müssen. Angetrieben durch eine Reihe von unter anderem idealistischen Motiven suchen Menschen wie er Möglichkeiten, durch eigene Aktivitäten wie auch durch den Aufbau von Kooperationen die Vermarktung in Gang zu setzen und Produktionsketten aufzubauen.

Für alle drei Gründungen ist die Vernetzung in ökologisch orientierte Kreise klar erkennbar. Dabei sind die Unterstützungsstrukturen zwar jeweils unterschiedlich, aber in allen Fällen vorhanden.
In keinem der Fälle scheint die langfristige Existenz der Gründung ohne das politisch-ökologische Umfeld denkbar.

\section{- Konsequenzen für die Gründungsförderung}

Grüne GründerInnen setzen bedeutende Impulse in der Entwicklung und Markteinführung von ganzheitlich in der Produktlinie optimierten, ökologischen und nachhaltigen Produkten. Sie leisten damit einen Beitrag zum nachhaltigen Wirtschaften, der bei der Analyse der Wirkung von Umweltmanagementsystemen in konventionellen Unternehmen noch zu oft vermisst wird. Dieser besondere Beitrag Grüner Gründungen zur Nachhaltigkeit macht die Förderung Grüner Gründungen umweltpolitisch wünschenswert. Aber die Förderung Grüner Gründungen ist nicht einfach. Grüne GründerInnen sind in zweifacher Hinsicht Ausdruck von Kultur:

- Zum Ersten sind sie Ausdruck einer Kultur der Selbständigkeit. Ausdruck dieser Kultur ist eine hohe Wertschätzung von Unabhängigkeit und Handlungsfähigkeit. Die Anerkennung, die selbständig Tätigen in Deutschland zukommt und die Fähigkeiten zur Selbständigkeit, die jungen Menschen vermittelt werden, bestimmen das Ausmaß, das Gründungen erreichen können. Trotz der allgemein guten und Gründungen begünstigenden Infrastruktur gibt es hier aber eine Reihe von Hindernissen, die ein rasches Anwachsen der Zahl der Selbständigen über die gegenwärtige Quote von etwa 7,5 Prozent aller Erwerbstätigen nicht erwarten lassen.

- Zum Zweiten sind sie Ausdruck sowohl der abklingenden Umweltbewegung als auch einer sich langsam entwickelnden Kultur der Nachhaltigkeit. Ausdruck dieser Kultur sind spezifische Werte, unter anderem eine hohe Wertschätzung der intakten Natur und des Umweltschutzes sowie intakter Sozialbeziehungen, eine kritische Einschätzung zur Möglichkeit technischer Lösungen von Umweltproblemen und eine auf Erhaltung und Reproduktion von Ressourcen orientierende, haushaltsökonomische Rationalität. An der Existenz Grüner GründerInnen wird deutlich, dass es eine Personengruppe gibt, die in der Lage ist, aus dieser Kultur heraus Gründungsideen zu entwickeln und Unternehmensgründungen zu initiieren. Diese Gruppe dürfte allerdings nicht sehr groß sein. Grüne GründerInnen haben in den letzten $30 \mathrm{Jah}$ ren sowohl die Veränderung der Lebensmittelbranche zur Nachhaltigkeit weit reichend mit beeinflusst und auch im Sektor regenerativer Ener- gien einen erheblichen Einfluss ausgeübt. Aber Grüne Gründungen sind nicht für einen - kurzlebigen - Boom gut, wie dies die Gründungen der Dotcoms oder der Biotech-Unternehmen waren. Grüne und nachhaltige Gründungen müssen aus ihrer Kultur heraus den Anspruch langfristiger Gültigkeit ihrer Lösungen entwickeln.

\section{Fazit}

Eine auf Nachhaltigkeit orientierte Wirtschaftspolitik und eine auf Nachhaltigkeit orientierte Umweltpolitik werden gut daran tun, die Förderung von Grünen Gründungen mit in das Spektrum politischer Instrumente zur Förderung der nachhaltigen Entwicklung aufzunehmen. Neben der Ordnungspolitik, der Produktpolitik und den ökonomischen Instrumenten der Umweltpolitik liegt hier eine zusätzliche Chance. Hier geht es nicht nur darum, das Bestehende ökologisch effizienter zu machen, sondern es geht um die Verbreitung der Kultur der Nachhaltigkeit. Es geht darum, bestehende Lösungen umfassend zu hinterfragen, neu zu denken und dabei konsistente, die Einheit von Produktion und Reproduktion möglich machende Lösungen zu finden. Und es geht darum, als Ausdruck einer Kultur der Selbständigkeit, selbstbewusste unternehmerische Impulse einer nachhaltigen Wirtschaftsweise zu setzen (4).

\section{Anmerkungen}

(1) Vgl. Clausen, J: Umsteuern oder Neugründen? Die Realisierung ökologischer Produktpolitik in Unternehmen. Dissertation, Books on Demand, Nordersted 2004, Download: www.suub.uni-bremen.de

(2) Vgl. Boeker, W. P.: Organizational origins. Entrepreneurial and environmental imprinting at the time of founding. In: Caroll, G. R.: Ecological Models of Organizations. Ballinger Publishing. Cambridge MA 1988, S. 33-51.

(3) Siehe auch Petersen, H.: Sustainable Champions. Positionierung von Markfführern im Umweltbereich. Eine empirische Untersuchung. Centrum für Nachhaltigkeitsmanagement e.V., Lüneburg 2002.

(4) Zur Vertiefung des Themas siehe auch den geplanten Schwerpunkt in Heft 2/2005.

\section{Der Autor}

Dr. Jens Clausen ist Wissenschaftlicher Mitarbeiter im Forschungsfeld ökologische Unternehmenspolitik am Institut für ökologische Wirtschaftsforschung (IÖW). Kontakt: IÖW Hannover, Hausmannstr. 9-10, 30159 Hannover. Tel. 0511-1640342, Fax 0511-164039, E-Mail: jens.clausen@hannover.ioew.de 
(c) 20I0 Authors; licensee IÖW and oekom verlag. This is an article distributed under the terms of the Creative Commons Attribution Non-Commercial No Derivates License (http://creativecommons.org/licenses/by-nc-nd/3.o/), which permits unrestricted use, distribution, and reproduction in any medium, provided the original work is properly cited. 